\title{
Máquinas en el aire: manifestaciones atmosféricas ${ }^{1}$
}

\section{Machines in the Air: Atmospheric Manifestations}

\author{
Agustín Mercado Reyes \\ Universidad Autónoma Metropolitana, Unidad Cuajimalpa | México \\ Contacto: agustin_mr@ciencias.unam.mx
}

\section{Resumen:}

El presente texto reflexiona acerca del concepto de atmósfera, en el contexto de la crisis climática actual, cuya complejidad se exacerba por su carácter global y urgente. Si bien dicha noción se puede entender a través de datos cuantitativos resultantes de la medición sobre un objeto corporal, la ontología que Félix Guattari desarrolló en sus últimos escritos nos provee de un sistema en el que cada entidad real presupone la convivencia de registros tanto corpóreos como incorpóreos, y tanto reales como posibles, y por tanto un entendimiento puramente corporal sería insuficiente. El sistema de Guattari invita a aceptar la multiplicación de las manifestaciones y de las potencias del objeto atmosférico, y señala la dificultad de articular y conciliar distintos tipos de valores inherentes que presenta.

Palabras clave: atmósfera, crisis ecológica, universos de valor, corpóreo e incorpóreo

\section{Abstract}

The present communication is a reflection on the concept of atmosphere in the context of the current climate crisis. This complex notion can be understood through quantification and measuring of a corporeal object; however, as Félix Guattari has shown in his late writings, every entity presupposes both a corporeal and an incorporeal side, and is a manifestation of possible and real ontological registers. As such,

\footnotetext{
${ }^{1}$ Texto realizado con el apoyo del proyecto CONACyT A1-S-21700: "Exploración de Creatividad Relacional No-Antropocéntrica”.
} 
a purely corporeal understanding of any object would be insufficient. Thus, Guattari deploys a thought that invites us to accept the manyfold manifestations and potencies of any object - including the atmosphere - and reveals the difficult enterprise of articulating different systems of value inherent to any object.

Keywords: atmosphere, ecological crisis, universes of value, corporeal and incorporeal

\footnotetext{
"We are like the dreamer who dreams and then lives inside the dream. But who is the dreamer?"
}

Twin Peaks: The Return. Part 14

\section{I}

La seguridad que brinda el apoyo conceptual es casi fisiológica. Hay, sin duda, mentes para quienes lo ideal sería que cada concepto fuera expresado de manera tersa y perfectamente pulida, y que cuyo despliegue y uso pudiera transcurrir sin elementos de fricción. Al lograr conceptos totalmente definidos y determinados, la realidad puede ser comprendida y catalogada de manera eficiente; los pasos del pensamiento se podrían dar con una vacilación mínima, engarzando un razonamiento tras otro. Existe un atractivo adicional: el entendimiento de la realidad a través de una epistemología formada por conceptos delimitados otorga, a su vez, una guía para actuar que, si no infalible, por lo menos se puede establecer como justificada.

Sin embargo, la organización de la experiencia no necesariamente tiene que ser un esfuerzo de delimitación. Si éste es el único objetivo de la conceptualización de la realidad, los conceptos posicionados en su propia parcela de distinción y la lógica que de ellos se sigue, tienen un efecto de corte, de atomización. Tal como lo ha expresado William James, los conceptos, al estar formados como definiciones cerradas en aras de la claridad intelectual, funcionan como un asidero al cual la lógica se aferra incluso más allá de su utilidad. Un concepto acabado y determinado hasta sus últimas consecuencias es una mediación frente a la experiencia. En su abstracción, un concepto no sólo pretende establecer positivamente lo que algo es, lo cual es un movimiento de por sí limitante; también indica aquello que ese algo 
no es, las negaciones que la mente debe de postular acerca del objeto que trata de comprender. Al abismo de esta negativa se arrojan el cambio, el flujo, la temporalidad, la comunión, la multiplicidad y la creatividad (James, 1987: 729).

Existe otro modo de conceptualizar, que trata de restituir la continuidad de la realidad en la experiencia. No se trata de negar el uso de conceptos, empresa que suena imposible en la mayoría de los decursos del pensamiento, sino de levantar el telón de la definición y observar qué tipos de experiencia se mueven tras de él. Necesariamente especulativo, el movimiento de aceptar conceptos que intenten reflejar la realidad es riesgoso; el riesgo mayúsculo, al menos desde mi punto de vista, es que la complejidad con la cual se quiere hacer una correspondencia ocasione un vértigo y una parálisis, la frustración de no hacer calzar exactamente las piezas de nuestros pensamientos como deberían, o el fracaso de encontrar un hilo dorado que indique exactamente cómo se tiene que desplegar una acción cautelosa.

El centro argumentativo de este texto es la concepción de atmósfera, y el objetivo es considerar las implicaciones de que el concepto de atmósfera —el cual se podría configurar de manera puramente física - se engrane de tal forma en una serie de elementos ontológicos que lo rebasen, que se mueven en registros disonantes. Las conexiones que traza no son exclusivas del concepto de atmósfera, pero en él se expresan de manera particularmente intensa; esa intensidad es un punto obligado de meditación, dada la importancia de los eventos que se juegan en nuestra época. No es inusual que un concepto tienda líneas en distintos registros y su aplicación se determine poco a poco, a través de su papel en un discurso. Esto no es lo mismo que una determinación a través de una definición, pues la definición de un término en un sentido operativo lo establece y lo posiciona para ser comprendido en un sistema con un fin determinado; por ejemplo, proveer una definición operativa que permita su ingreso en un estudio científico.

Una metaforización, una metonimia, el rescate de una palabra cotidiana, un decurso etimológico: todos estos procesos pueden dar origen al nombre de un concepto, como lo señalan Deleuze y Guattari (2005: 13). Por ejemplo, Anderson (2009) desarrolla un concepto de atmósfera derivado de un pasaje de Marx, en donde se mezclan características puramente físicas con sensaciones y emociones: no sentimos, decía Marx en 1856, la fuerza opresiva de una atmósfera revolucionaria, así como no detectamos en ningún momento la atmósfera de la Tierra que ejerce sobre nosotros un peso de 20000 libras. La noción de "atmósfera", inscrita en el llamado "el giro afectivo", invoca una "imaginación material" para pensar en "atmósferas afectivas": un sustento de las sensaciones, espacialmente difuso, que emerge de personas, lugares u objetos. Es posible pensar en una atmósfera serena, melancólica, deprimente, erótica, sagrada. En efecto, es posible hablar de la atmós- 
fera de una biblioteca y su concreción a través de los olores de papel y madera, del silencio y del significado social del espacio; pero deseo permanecer en lo que podríamos llamar el concepto puramente meteorológico o geológico. Y el objetivo es mostrar que una noción fundamental para el pensamiento climático no puede evitar retomar y actualizar elementos incorpóreos; que lo atmosférico en sentido meteorológico es en sí lo suficientemente problemático, no sólo para el establecimiento de políticas públicas y guías gubernamentales, sino de una acción y pensamiento personal.

Aquí, entonces, no trato de seguir los afectos, sino intento pensar acerca de las afecciones, de las manifestaciones indirectas del objeto que las causa. La cuestión central aquí, no es tomar un concepto y buscar sus diversas apariciones en distintos contextos; sino argumentar que si existe un objeto de interés del cual conocemos datos, dichos datos deben de haber emergido de cierta configuración del objeto en un territorio conceptual. En tanto que esa configuración nos rebasa, es posible que la manera más apropiada de acercarse a ese objeto sea un acercamiento especulativo y descentralizado, en donde ni el objeto se agota en los datos que nos ofrece, ni se constituye como algo determinado por la simple definición.

\section{II}

Inhalar y exhalar: esta acción se realiza sobre un sustento material dado, tan disponible que en condiciones usuales requiere de una atención exclusiva para registrarla. No se necesita mucho más que esta acción, compleja pero cotidiana, que revela el carácter doble de la atmósfera, simultáneamente decidible e indecidible de manera física. Inhalar y exhalar: el ejemplo perfecto para la experiencia inmediata, el paso primordial para la subsecuente contemplación de un objeto. En sus inicios, la noción de atmósfera habla de algo que está totalmente dado como una experiencia viva y de la vida; en palabras de Alexander (1966: xxxviii): "dado el aire, yo respiro; dada mi respiración, hay aire". Pero inhalar y exhalar, en tanto que experiencia constitutiva de la vida al grado de confundirse con la propia duración vivida, perfila la dificultad de la determinación total de un concepto, aunque éste sea técnico o científico. ¿Cómo delimitar la continuidad del aire con quien lo experimenta y por tanto lo conceptualiza? El experimentar —y más de manera inmediata- a través de un evento de duración establece una conexión profunda y no trivial entre aquello que está experimentando y aquello que está siendo experimentado. La conceptualización intenta dar un paso atrás; pero la experiencia inmediata de la respiración no se detiene nunca, y en ese caso particular difumina la separación 
que se establece no únicamente entre quien concibe y quien es concebido, sino entre el punto de concepción y el punto de experimentación (de "disfrute", diría Alexander) que informa la concepción y se posiciona simultáneamente como una antípoda y como un punto de generación. La definición de la noción puramente meteorológica se deriva, desde un primer momento, de esta experiencia inmediata de la atmósfera: la intuición de un gas en contraste con otros estados de la materia. El glosario del último reporte de evaluación (AR5) del Panel Intergubernamental sobre el Cambio Climático (IPCC, 2013), consigna el concepto de atmósfera como "el envoltorio gaseoso que rodea a la Tierra". En cuanto definición operativa, es determinada de manera termodinámica, pero vale la pena enfatizar que es una definición tan intuitiva que puede ser realizada visual o táctilmente. El camino para establecer líneas divisorias claras sería una red de recortes que parece ser artificial: un recorte espacial (establecido, ¿dónde? ¿en los alvéolos? ¿en la nariz?) y uno temporal (¿dictado exactamente por qué proceso?). ¿Cómo podríamos lidiar, por ejemplo, con el recambio químico del gas antes y después de la intervención fisiológica de la respiración?

Aunque es elemental en el imaginario, la manifestación física de la atmósfera es fluida. Es aparentemente separable de lo terrestre y al mismo tiempo resistente a un corte definitivo. Este efecto se replica en todas las escalas temporales y espaciales; por ejemplo, Whitehead (1920: 167) nos invita a pensar en un objeto sólido, como un monumento de piedra (el Obelisco de Cleopatra), y nos recuerda que su solidez y la posibilidad de separación de su entorno, es únicamente una abstracción; a lo largo de diversos lapsos temporales, la "danza de los electrones" hace que la piedra misma se confunda con la atmósfera, tomando materia de ésta y liberando a su vez partículas al aire. El dinamismo que problematiza la distinción entre la atmósfera y lo demás, se trasluce en nociones básicas de las ciencias atmosféricas. Por ejemplo, la zona límite (boundary layer) de la atmósfera es la zona inferior de la tropósfera en donde se presentan turbulencias derivadas de la fricción con la tierra; es una zona limítrofe, estructurada pero dinámica, y sujeta a cambios tanto espaciales como temporales. En el día es más amplia y en algunas regiones (como desiertos) potenciada por sistemas de convección; en la noche, se vuelve casi estática, y su grosor es mínimo. En promedio, comprende el $10 \%$ de la tropósfera (aproximadamente un kilómetro), y a su vez, el $10 \%$ de esta zona límite es la capa superficial, en donde el intercambio de energía y materia es mayor, y que funciona como amortiguador para mantener estáticas las capas altas de la atmósfera (Cushman-Roisin, 2019).

El corte de la atmósfera a través de una capa superficial, en donde ocurren la mayoría de los intercambios con "lo demás”, es simplemente una manera conven- 
cional — signa data — de intentar dotar nuestros análisis de sentido y significado a pesar de la dificultad de englobar el objeto en el concepto. Inmanejable en su enormidad, notablemente resistente a la cartografía, las características atmosféricas sugieren que, en contraste con lo anterior, su teorización tendría que ser menos convencional, y no tomarla como un objeto completamente discernible. Por ejemplo, considerar la atmósfera como un hiperobjeto (Morton, 2013) significa pensarla como un objeto que es, de alguna manera, más que un objeto, como una entidad tan masiva que su propia constitución deriva en efectos no locales, en una presencia en dimensiones parcialmente ajenas a lo humano, que le otorga una cualidad caleidoscópica. Posiblemente sea más apropiado pensar en la atmósfera como algo profundamente indeterminado, cuya conceptualización misma ha pasado por una serie de convenciones de delimitación, que físicamente interfiere con todo lo que está en contacto con ella, y no como un envoltorio o como un medio inerte, fácilmente determinable, como "la capa gaseosa que envuelve a la Tierra". Las zonas atmosféricas tienen una lógica propia que rebasa una estructura fácilmente enunciable. Una síntesis global es profundamente difícil, pues sus características medibles están distribuidas de manera desigual. Por ejemplo, la humedad es particularmente difícil de establecer tanto descriptiva como predictivamente, pues, en cualquier momento dado, existen zonas de alta humedad, inmediatamente adyacentes a zonas extremadamente secas; de igual manera, los cambios en el clima presentan una estructura diferencial a lo largo de la atmósfera. Para aumentar todavía más la complejidad, los datos actuales solamente abarcan la tropósfera, pues las mediciones de más altitud son muy inexactas y variables, sobre todo en la estratósfera (IPCC, 2013: 196, 232).

Las cualidades de interpenetración y diversificación de efectos de la atmósfera se ven intensificadas por su papel protagónico en el calentamiento global, el evento ambiental más urgente y más visible de nuestros tiempos, que se asocia directamente a otras crisis como la pérdida de biodiversidad, la creciente desertificación de varias regiones del planeta, el próximo punto de quiebre en términos de cosechas productivas, la dependencia actual de la actividad de producción en procesos contaminantes, entre otros. El calentamiento global está dado, en última instancia, como un calentamiento atmosférico. A partir de ese punto, el problema se abre hacia un número indeterminado de efectos: el calentamiento de los mares, la fusión masiva del hielo en distintos puntos del planeta, la liberación de más gases de invernadero por el descongelamiento del permafrost, la alteración de los ciclos hídricos; pero, en primer lugar, la causa primaria es la acumulación de gases, generados por procesos antropogénicos. En las comunicaciones técnicas especializadas se establecen medidas escalares fácilmente comparables: la temperatura de la atmósfera en 
los niveles preindustriales de dióxido de carbono; la temperatura actual, cuya dinámica ha descrito una tendencia ascendente, y la temperatura dentro de diez o treinta ańos, que informa las predicciones acerca del destino del planeta completo. ¿Cómo es posible, frente a la heterogeneidad atmosférica, hablar de mediciones de temperatura de la atmósfera?

Para dotar de sentido a los datos, hay procedimientos que no tienen análogo en la mayoría de las ramas de la ciencia, que son requeridos debido al número y a la heterogeneidad de instrumentos de medición de parámetros atmosféricos, y al diluvio de datos que ellos producen. Es una tarea de contraste y conciliación de datos. Por ejemplo, es necesario contrastar la invaluable y antigua serie de datos producidos por estaciones climáticas terrestres con los datos producidos por las nuevas tecnologías, tal como el mapeo de columnas tridimensionales registradas por satélites. Aún más, la serie de tiempo está en constante re-análisis, pues uno de los resultados más importantes es la evolución de la temperatura global. El re-análisis no es únicamente el descubrimiento de registros antiguos o la producción de registros nuevos; más bien, constantemente se realizan simulaciones nuevas: se vuelve a reproducir la cinta de la evolución atmosférica tal como lo entiende la computadora, por decirlo así. Cada nueva simulación arroja un rango esperado de error que nos informa la certeza de nuestros datos imperfectos, propensos a errores o, a final de cuentas, registrados por objetos locales para tratar de entender un hiperobjeto no local. ${ }^{2}$ Así, nuestros datos de la complejidad atmosférica sirven para construir modelos que nos ayudan a verificar la certeza de nuestros datos, en un ciclo de retroalimentación constante (Lovejoy, 2017; IPCC, 2013: 185); por tanto, la descripción científica de la atmósfera ni siquiera está asociada a un objeto plenamente presente: difumina sus alcances tratando de re-analizar continuamente el pasado, intentando volverlo a simular, inventando nuevamente la noción de atmósfera desde cero, partiendo de los datos que tenemos pero con miras a resignificar esos datos. En palabras de Edwards (2010: 336): "El re-análisis ha sido integrado a la infraestructura del conocimiento climático. Cada iteración traerá consigo nuevas revisiones a la historia del clima. Incluso en el futuro lejano, seguiremos re-analizando el pasado: más imágenes globales, más versiones de la atmósfera, todas ellas brillando dentro de una franja relativamente estrecha, pero nunca estabilizada en una línea definitiva”.

Desde luego, esto no pretende poner en duda una de las certezas más firmes de los análisis climáticos: que el mundo se ha calentado gradualmente, por efectos de la actividad humana, desde el siglo xIX y de manera vertiginosa y cada vez más

${ }^{2}$ Acerca de las fuentes de incertidumbre de los datos, es posible consultar IPCC (2013: 165). 
acelerada desde hace 50 años. El problema aquí no reside en una disputa ni una disociación, sino en una articulación: si lo corpóreo es de por sí profundamente complejo e inasible, ¡cómo es posible pensar esa heterogeneidad corporal al lado de otras heterogeneidades inherentes al objeto? ¿Cómo pueden emerger valores en cada uno de estos terrenos heterogéneos, y cómo es que estos valores se comunican entre sí?

\section{III}

La noción científica de atmósfera se problematiza a sí misma; se envuelve a sí misma en complicaciones de determinación y en intentos de establecer un carácter discreto. Sin embargo, hemos de notar que únicamente hemos tocado una parte superficial de las potencialidades ontológicas, el mero dominio temporal-espacial. Es posible extender aún más este panorama de la ontología; pues si el intercambio de energía y materia es importante, el sistema que proponemos seguir es tal que considere las manifestaciones que no son corporales (aquello que Spinoza agrupaba en el atributo general de "pensamiento") como inmanentes a lo real. Por ello, una posición materialista que "determine como su punto de partida la existencia de un mundo material que sea independiente de la mente", como lo propone DeLanda (Dolphijn y van der Tuin, 2012: 39), dejaría fuera gran parte de lo que aquí resulta problemático y productivo, a pesar de su énfasis histórico. Es decir: si la experiencia de inhalar y exhalar desmonta la delimitación clara entre la atmósfera y lo demás, la experiencia de difusión conceptual que así se logra está limitada a un intercambio de materia y de energía. En pocas palabras, es un Flujo corpóreo determinado, el cual se puede seguir y descomponerse hasta la visión microscópica del destino de cada molécula (lo cual implicaría una multiplicación intratable de la información), o se puede condensar en una discursividad global (a través del procesamiento de los datos con ayuda de modelos). Incluso considerando esta diversidad, sin embargo, el pensamiento a través de Flujos permanece en un terreno puramente corporal.

La noción de "Flujo" es una de las cuatro partes del aparato de pensamiento que Félix Guattari desplegó en los últimos años de su vida, para ir más allá de la corporización de la realidad. La parte corporal (representada por las partes de los Flujos reales y los Phylums posibles) adquiere gran parte de su poder y de su uso a través de una lógica binaria, "en una oposición binaria con un fondo" (Guattari, 1996: 43). Para intentar pensar con libertad, Guattari multiplica los puntos de articulación cuyas potencias posee la realidad, para entender la riqueza ontológica 
cuyo fin explícito es "hacer un mapa de las configuraciones de subjetividad [...] sin tener que recurrir a los aparatos tradicionales de infraestructura somática" (léase: corpóreo), "determinismo, condicionamiento conductual, etc.” (Guattari, 2013: 26-27). Como contraparte de lo corpóreo, Guattari postula dos figuras incorpóreas y virtuales: los Territorios existenciales reales, y los Universos de valores posibles. ${ }^{3}$ Superficialmente, su objetivo parece ser la comprensión de un sujeto humano; pero no hace falta más que ver sus obras inmediatamente posteriores — pienso en Les Trois écologies de 1989 y Chaosmose de 1992_ para que se deje ver la línea subterránea a la que apunta su pensamiento: una subjetividad no escapa únicamente a la necesidad de ser individual, sino a la necesidad de estar referida exclusivamente a lo humano. Esto no quiere decir, por otro lado, un abandono de la subjetividad humana como parte constitutiva de la realidad. El énfasis en lo no humano no es "anti-humano", no es una especulación contrafáctica en cuyo centro está la ausencia de lo humano. Al contrario, como en la obra de Deleuze, la inmanencia atraviesa sin tregua el pensamiento de Guattari. La pertenencia de lo humano a la realidad es indudable, y el pensamiento especulativo de Guattari no pretende negar ese hecho básico. Los Territorios existenciales y Universos de valor no intentan hacer desaparecer la subjetividad humana, sino descentrarla. Aquí, por tanto, la especulación no se trata de establecer contrafácticos, la multitud de los cuales es resumida y condensada en la categoría de Phylum, que comprende las posibilidades de lo actual no realizadas pero siempre presentes como un lado tendiente al infinito en número. Siguiendo la recomendación de Stengers (2018: 29), la especulación más bien tendría que ser tomada entre las manos, evaluada en términos de los problemas que levanta, de las experiencias con las que se piensa y de los efectos que genera.

El establecimiento de un pensamiento, a través de sistemas actuales pero no realizados, es una manera de pensar en donde se insiste en la capacidad de lo actual para generar más y más alternativas de manifestaciones posibles. Los Flujos de materia y energía pueden configurarse de diversas maneras y establecer una historia distinta de sus propias interacciones, dando lugar a una infinidad de es-

${ }^{3} \mathrm{El}$ sistema combinatorio de cuatro partes generales es desarrollado en Cartographies Schizoanalitiques (2013). Resulta particularmente interesante comparar la noción de Guattari de un "virtual posible" y un "actual posible" con diversos pasajes de Deleuze en donde por un lado explícitamente opone lo posible y lo real, y por otro insiste en que lo virtual es plenamente real. Esto sugiere que lo posible, como concepto, es cambiante en la obra de Deleuze, desde ser un estado isomórfico a la realidad que simplemente no tiene la cualidad de ser real, hasta volverse una fuerza creativa (Deleuze y Guattari, 2005: 168), aún en oposición con lo real pero fundamental tanto en el dominio estético como en la creación de nuevos modos de vida posibles. 
tados. Pero incluso frente a esta posibilidad, toda manifestación (real o posible) de lo actual se establece en el campo de los "muchos", de lo múltiple, sin transmutarse en una multiplicidad. Lo múltiple establece, por necesidad, la noción de una lógica de exclusión, en donde queda demarcada mi propia frontera que me delimita de lo demás. En términos lógicos, esto establece un terreno claro y natural: "yo soy yo". Sin embargo, si algo se intentó dejar en claro en la sección anterior es que la aplicación de este pensamiento a todo lo que llamamos "realidad" —y, en su versión más estricta, pretender aplicarlo en todos niveles—, implica un ejercicio de abstracción. Paradójicamente, la separación con la que me concibo como algo distinto de mi medio está fundada en una lógica de fusión: "yo" me compongo de muchas partes que necesariamente tienen que fusionarse estableciendo una continuidad perfecta (pero interna) para establecer la integración necesaria y "secretar una delimitación relativa" (Guattari, 2013:59) con lo demás, con lo externo.

La manera de experimentar las manifestaciones corporales, sean éstas actuales o posibles, se lleva a cabo a través de datos, sea cual sea la forma o el formato que estos presenten; no únicamente datos sensoriales, sino también datos numéricos, extrapolaciones, aproximaciones, parámetros; data, en general. Según Guattari (2013: 58), si hay algo dado (donné), hay también algo que lo da (donant). La separación poco o nada tiene que ver con una noción puramente trascendental. En efecto, desde cierta óptica es posible decir que no hay separación dada entre ambos registros. Guattari sugiere que aquello que da está marcado por un grasping y refiere, de pasada en un pie de página, que ese anglicismo lo ha recogido de la filosofía de Alfred North Whitehead. Antes de poder dar datos, antes de funcionar como donant, cualquier cosa real debe tener un agarre sobre algo para la constitución de su propia existencia, un grasping, aunque sea autorreferente. Esto es una parte fundamental del proceso de concrescencia de Whitehead, en donde la entidad actual retoma todo lo que hay en el universo para incorporarlo a su propio ser. Esta es una experiencia pura, para sí, pre-individual, tal como la propone James (1996) con el término de "experiencia pura", un nivel accesible sólo por especulación. Con ese pie de página, entonces, Guattari nos deja ver sus cartas: la distinción de sujeto y objeto no puede funcionar como una polarización en donde uno está estructurado por el otro, sino que son partes necesarias, heterogéneas de la complejidad inherente a cada punto de la realidad. Sin embargo, antes de hablar de una "muerte del sujeto" o una "superación de la dicotomía sujeto-objeto", Guattari (1996: 40) apunta a una subjetividad distinta una subjetividad "pática", en sus palabras, que intenta simultáneamente ir "más allă" y "más acá" de la división tajante de sujeto y objeto: liberar la noción de subjetividad de las restricciones de lo humano, 
pero también hacer énfasis en los componentes pre-individuales que son necesarios para la constitución de cualquier elemento de la realidad.

Los puntos generales de esta subjetividad extendida, según Guattari, son la creatividad radical; la parcialidad inevitable; la pérdida de límites, tanto en el sentido de delimitación de un otro como en el de una finitud de potencias; la constitución a través de una heterogeneidad irreducible, y -en mi opinión, una de las características que cristalizan la dificultad del pensamiento en y con estos sistemas- una permanencia de los elementos heterogéneos en la complejidad de la mezcla. Y en efecto es una mezcla: una mixis en el sentido estoico, en el que todo puede ser penetrado, todo puede interconectarse, nada es indivisible y cada elemento puede abandonar la mezcla dadas las condiciones correctas, "como la mezcla de agua con aceite", según Schuhl (1962: xxi), de quien Guattari toma el concepto. Es por ello, continúa Schuhl, por lo que un soplo (un souffle) puede interpenetrar todo obstáculo. Los componentes de la realidad, corpóreos e incorpóreos, actúan bajo un principio de conexión íntima, y se afectan "tal como el incienso se expande en el aire" (1965: xxi). Considerar a lo subjetivo como una mezcla afirma constantemente que la formación de subjetividad es algo necesariamente colectivo, pero es también imposible una reducción de la formación de la subjetividad a lo meramente social. La subjetividad se extiende, según Guattari, "al campo social, al campo tecnológico [...] rebasa totalmente la esfera antropológica, y se extiende al devenir-animal, al devenir-planta. La subjetividad es lo que es lo más rico, lo más heterogéneo. Decir que está en el campo social, eso es de por sí reduccionista” (Guattari, 2011: 41).

La insistencia en una inmanencia radical libera a la subjetividad de su encierro en un domino plenamente antropocéntrico. Podríamos aventurar otra serie de engranes que constituyen el presentimiento de esa subjetividad liberada del modelo preconcebido de lo humano:

1. En cuanto proceso, la subjetividad no tiene estabilidad como algo delimitado, con respecto de lo demás. El proceso de conformación de cualquier objeto de la realidad — por ejemplo, nuestro objeto central, la atmósferaes una actividad de incorporación de lo demás, a tal punto que la barrera entre el objeto y lo demás se vuelve completamente propensa a ser atravesada. La atmósfera, en tanto que fluida, es un modelo perfecto para pensar este fenómeno. Si se quiere pensar a través de la bella imagen de Guattari, estos nodos de subjetividad componen una polifonía, el efecto sonoro en el que un sonido adquiere un excedente a través de su convivencia con otro sonido. Tercera mayor, tercera menor, cuarta aumentada: 
todos esos elementos polifónicos ponen en primer plano la visión doble entre un centro de constitución y otro, pues no podemos decir que el sonido pierda su identidad, pero tampoco podemos negar que esa identidad está constituida en parte importante por lo demás.

2. Un centro en donde una subjetividad se constituye sufre experiencias, nacidas de la convivencia con algo otro. Sin embargo, la experiencia no es nunca estable, ni siquiera en su propia identidad. En relación a sí misma y al mundo en que necesariamente se mueve, la experiencia (y por tanto, la subjetividad por ella constituida) constantemente cambia de énfasis, de marcos de abstracción; en una palabra, de valores. En el lenguaje de Guattari, se configuran, constantemente nuevas constelaciones en Universos de valor. Ésta es la faceta de interés central en el presente texto.

3. El carácter fluido y cambiante de los nodos de subjetividad — tal como se tratan aquí - trae a cuenta la característica más misteriosa, y la que merecería un pensamiento más detallado en una reflexión futura. Si todo está constantemente cambiando, si la incorporación de lo demás disuelve la identidad monolítica, si cada cosa está expuesta a la mutación de valores que a su alrededor se mueven, entonces todo es profundamente frágil. El inconcussum quid que se ha buscado para establecer la subjetividad de manera firme es, simplemente, una ilusión o una abstracción. La eternidad, según Guattari, es una infantilización inaceptable. ${ }^{4}$ Todo es finito, y la presente idea de subjetividad es una invitación a lidiar con ello.

Estos tres elementos (no exhaustivos, ni mutuamente excluyentes en su concepción) sirven para enfatizar que desde esta visión, los Flujos de la materia y energía y su evolución posible en tanto que Phylum son fundamentales; son los puntos abstractos en donde la materialidad se puede expresar plenamente; pero de ningún modo cuentan toda la historia de la realidad. Requieren una contraparte que pueda convertir un mero estado de las cosas (sea la conjunción de cuerpos sólidos que se atraen unos a otros, sea un estado de un sistema nervioso explicado con impulsos eléctricos, sea una visión de un ecosistema como un flujo trófico o fisicoquímico) en un evento, en un punto en donde un mismo estado de las cosas pueda reconfigurarse espontáneamente a través de diversos marcos de énfasis y de

4 "Esta adversidad no tengo solamente que aceptarla, sino también amarla por sí misma; tengo que buscarla, dialogar con ella, excavarla, ahondarla. Es ella la que me hará salir de mi narcisismo, de mi ceguera burocrática, la que me restituirá un sentido de la finitud que toda subjetividad mass-mediática infantilizante se dedica a ocultar" (Guattari, 2015: 389). 
ilimitadas interacciones de sistemas de valor, entre los cuales los valores antropogénicos son simplemente una fracción de la riqueza infinita de lo real.

¿Es posible decir, a través de esta descripción somera del entendimiento de la subjetividad, que la atmósfera puede considerarse como tal? ¿Es acaso un punto de demasiada fricción conceptual dotar de algo como nuestra subjetividad a lo atmosférico? Tal vez, sin embargo, mi argumento es que ese objeto fluido, interpenetrante e interpenetrable que llamamos la atmósfera, revela de manera evidente las cualidades de constitución subjetiva que recorren la obra tardía de Guattari. No es de sorprender. Él mismo propone constantemente una guía de acción frente a las crisis actuales que debería de considerar tres registros: el mental, el social y el medioambiental, como singularmente ecológicos. La idea de una subjetividad ambiental, complementaria a una ecología mental o social, necesariamente obliga a un ejercicio constante de especulación. Y si nombrar al fenómeno atmosférico como un nodo de producción de subjetividad resulta insatisfactorio, podemos usar la puerta de escape del propio Guattari: podemos hablar de protosubjetividad, si parece necesario preservar un dominio ontológico para lo antrópico. Pero si anexamos el prefijo "proto" por lo inacabado, por la cualidad de "siempre en proceso", debemos notar que no existe una subjetividad perfectamente acabada, un monolito de experiencia que se determine de una vez por todas: toda subjetividad, en cuanto compuesta por ingresos de alteridad, por bifurcaciones internas, por una incorporación de lo eterno en una existencia finita, sería un modo de protosubjetividad. No hay manera de elucidar una distinción binaria absoluta en ningún evento realmente real, sin caer en una abstracción, tal vez heurística, pero potencialmente destructiva.

\section{IV}

Hasta aquí se ha afirmado lo siguiente: el fenómeno atmosférico puede ser considerado como un proceso de subjetivación. Lejos de ser una tesis metafísica ociosa, es una idea cuyo papel principal en este momento es su propio despliegue frente a decisiones prácticas, frente a problemas tangibles y urgentes. Vale decir que la subjetivación que aquí se propone no es de ningún modo una especie de panpsiquismo; decir que la atmósfera disfruta o sufre en sí un proceso de subjetivación, no pretende asignarle ni una intencionalidad ni una conciencia. Es, más bien, señalar que el ingreso del proceso atmosférico coincide con las líneas de lo que Guattari (1989) llama una visión maquínica del ambiente: "se podría re-calificar a la ecología medioambiental de ecología maquínica, en tanto que, ya sea en el cosmos 
o en las praxis humanas, nunca se trata de otra cosa que de máquinas" (68). Máquina, en este sentido, es un concepto ontológico y no una categoría de producción antropogénica. Una máquina no debe ser entendida como una herramienta, como un objeto diseñado y usado por manos humanas para un fin determinado; no debe de ser entendida como algo artificialmente producido, ni debe de ser concebida como una repetidora de la misma función que determine su funcionamiento o su ruptura. Una máquina de Guattari, en fin, no es mecánica sino maquínica. Es una rebelión conceptual frente a la supuesta necesidad de una estructura, en la posición y el papel fijo de cualquier parte de esta máquina; aún más, es una llamada a reconsiderar la difícil imbricación de lo que puede ser considerado puramente material con una serie de valores no corpóreos. Tal es el pensamiento que he buscado en este corto texto.

Si la atmósfera es considerada una máquina en ese sentido, debe ser una máquina de potencias infinitas. La tarea de esta sección es únicamente explicar cómo puede entenderse esta infinitud. No es una idea que pueda surgir exclusivamente con la mirada contemporánea sobre la atmósfera; desde el principio del siglo XvIII, Leibniz batallaba con la idea de un infinito que no se pudiera entender como partes sumadas a las partes, en donde el número de partes no sea infinito, "porque un infinito no podría ser un verdadero todo" (Leibniz, 2017: 227). Tal infinitud no es una infinitud de partes anidadas una dentro de otra, como el Arlequín de la commedia que sale al escenario vestido con capas y más capas de ropa, sino que tiene que pensarse como poseedora de "una sutileza totalmente diferente" (Leibniz, 2017: 438). En un famoso e impactante pasaje de la Monadología, Leibniz compara esta infinitud de las máquinas "naturales" (en oposición a las "artificiales", que aquí podríamos llamar "mecanismos"), que existen en cada porción de la materia, con un jardín lleno de plantas, en el que cada rama de cada planta es a su vez otro jardín (en Strickland, 2014; \$66-68): cada parte de la realidad es un mundo lleno de mundos.

Ahora bien, no pretendo anunciar un vitalismo total, que corte a través de lo que entendemos como orgánico e inorgánico. Propongo continuar con la visión ontológica de Guattari, que intenta otorgar a Leibniz la "sutileza" que este último busca, la cual encontraremos en la apertura ontológica a los universos de valor, que exceden por mucho la descripción corpórea de cualquier punto de la realidad. En cada cosa y en cada momento, los mundos infinitos que se contienen no son partes extra partes aunque su identidad (siempre temporal, siempre con límites difusos) está implicada en diversos tipos de alteridad. Por ejemplo, aquella derivada de la consistencia material, pero también la alteridad que surge en la comparación de la máquina existente con su "diagrama", con la máquina abstracta que no se ha 
manifestado en una actualización pero que por ello es real como una serie infinita de potencias; la alteridad histórica del decurso evolutivo de la máquina, o la alteridad consigo misma, una alteridad que, llevada al extremo, tiende a autodestruir o a hacer colapsar desde dentro la propia máquina. ${ }^{5}$ Creo que en el año 2020 , el autoagonismo de esta última alteridad se ha expresado como nunca, y todo parece indicar que en los años venideros tendremos la oportunidad de ver más y más manifestaciones de este fenómeno.

En estos tonos del pensamiento maquínico de Guattari podemos observar el reflejo de una secuencia de argumentos con los que Gregory Bateson (1999) ${ }^{6}$ analiza lo que él llama "las patologías de la epistemología”. Según Bateson, existen varios pensamientos distintos, y varias epistemologías posibles. Muchas de estas epistemologías informan nuestras acciones; esto es normal. También es posible decir que muchas de ellas funcionan: nos sirven para llevar a cabo ciertos fines, para desarrollar ciertas actividades que de otro modo no serían posibles. Esas viejas defensas de la mentalidad científica, que insisten en la capacidad de la humanidad de, por ejemplo, poner a un ser humano en la luna, de poder coartar el desarrollo de enfermedades, o de conocer eventos más allá de las capacidades sensoriales humanas, se basan en un enfoque de función y aplicación. Sin embargo, la funcionalidad de estas epistemologías no es garantía de que ellas sean correctas; la funcionalidad es únicamente un valor posible entre muchos otros. Es necesario insistir, en un contexto de urgencia actual, que la valorización toma diversos aspectos, y la funcionalidad de la epistemología totalmente abocada a lo corporal no es garante de la universalidad de sus valores. En efecto, ese garante de universalidad no puede existir porque los valores que se desprenden de una epistemología siempre están asociados a un "Universo" particular: a un registro de virtualidad que debe de convivir con otros tantos. Existen Universos de valores con "constelaciones” propias: valores estéticos, epistemológicos, sociales, políticos, etcétera. Una noción de valor tal, en tanto que inmanente, se aleja de manera explícita de dos interpretaciones usuales. Por un lado, no son valores morales, sino éticos, tal como insistió Deleuze

\footnotetext{
${ }^{5}$ Según Guattari (1996: 54) una máquina abstracta se pone en relación con una máquina concreta heterogeneizando componentes de todo tipo: técnicos, sociales, cognitivos, corporales, científicos, informacionales; como abandona la axiomatización, se opone a la unificación y a la estructura, al tiempo que enfatiza los valores de la singularidad y la potencia no actualizada que siempre forma parte de la realidad. Así, un diagrama entendido como máquina abstracta es una virtualidad que se puede concretar, creativamente, en sus actualizaciones. Además, Guattari sugiere que dicho concepto refiere también a procesos no humanos - noción fundamental para el desarrollo del presente argumento.

${ }^{6}$ El reflejo es explícito: Guattari elige una frase de este texto como epígrafe a su libro Caosmosis (1996).
} 
a lo largo de toda su obra fuertemente teñida de spinozismo: son valores en perspectiva, cuya manifestación detectable es la composición o descomposición, y únicamente un conocimiento correcto hará que las interacciones desplegadas en el mundo hagan aumentar las potencias de aquello con lo que estemos en contacto. Por otro lado, tampoco son valores cuantificables, y mucho menos valores de uso o de cambio. Como lo expresa Brian Massumi (2018) para retomar y reclamar la noción de valor, ésta tiene que ser no normativa, disociada del valor económico (y, particularmente, del capitalista), pero conectada con una alteridad inmanente.

Los valores que emergen de la máquina atmosférica no pueden ser calificados de una manera unívoca. En la crisis actual a la que nos enfrentamos, las modificaciones que ha sufrido la atmósfera recientemente se podrían referir a la acción de "la especie humana", por lo que se ha inaugurado la era geológica del Antropoceno. Sin embargo, tanto en términos de responsabilidad, como de causalidad y de efectos, el calentamiento global es profundamente asimétrico. Los desastres naturales derivados del aumento de la temperatura atmosférica tendrán sus peores efectos en poblaciones de por sí vulnerables, como ha ocurrido hasta ahora. No hay un anthropos generalizado, sino un coro polifónico de voces de diversidad ilimitada, cada una de las cuales construye una historia dependiendo de valores particulares; por ello, el término mismo de Antropoceno ha recibido críticas de autoras como Haraway (2016) proponiendo pensar el cambio en términos de un Capitaloceno. A otra escala, sin embargo, los cambios ambientales han traído efectos en valores políticos: han hecho que estados completos movilicen un pensamiento que enfatiza la propia seguridad, por ejemplo, a través del acaparamiento de recursos hídricos frente a la sequía regional que promete traer el cambio climático. Ni siquiera la concepción del objeto atmosférico, sin considerar sus cambios recientes, escapa de haber sido producida a través de la confluencia de Universos de valores tecnológicos, epistemológicos, políticos, matemáticos e incluso emocionales y estéticos. Sólo habría que observar el origen de la ciencia de la meteorología, que fue una lucha para "purificar" el entendimiento atmosférico de valores que no fueran científicos. Desde sus primeras configuraciones a mediados del siglo XIX, naturalistas como Alexander von Humboldt y Charles Piazzi Smyth fueron figuras centrales en el gran esfuerzo para separar las emociones estéticas de las hebras del conocimiento "científico" de la atmósfera y sus elementos, con el fin de fabricar una ciencia "verdadera", separándola así del influjo casi inevitable de las "ideas" emocionales y privilegiando los datos sensoriales (Dry, 2019: 75). Esta plétora de valores no acaba en el nivel humano. Por ejemplo, el dióxido de carbono, principal gas de efecto invernadero, ha ocasionado no solo un aumento de la temperatura sino un reverdecimiento de la cubierta vegetal del planeta (Reiny, 2016). Los valores movi- 
lizados siempre se resisten a ser calificados totalmente mediante una aproximación simple, variando en distintas escalas temporales y espaciales, sobre las especies no humanas, y, diría Guattari, también sobre especies incorpóreas.

Paso a paso, intentamos problematizar las barreras entre una cosa (la atmósfera) y otras. Nunca será posible levantarlas; en cierto sentido es una ventaja, pues la disolución de barreras posiblemente implique, inevitablemente, un sistema en el que toda la realidad responda al mismo llamado homogeneizante. Incluso en términos mentales, desde nuestro sesgado punto de vista, no hay modo de olvidar el "yo soy yo" (o no lo es, al menos, en un funcionamiento habitual de la mente, sin perturbaciones específicas de la química neuronal, que en este momento no nos competen). Es necesario considerar el carácter de dichas barreras a través de una lente totalmente inmanente; el acto de equilibrio es precisamente buscar cómo las barreras entre un objeto y la alteridad se pueden reconfigurar como articulaciones, es decir, como relaciones cuya condición necesaria es funcionar simultáneamente como distinción y unión, como contraste sin separación total. Es por eso que me atrevo a pensar en un registro distinto a las versiones del materialismo que insisten en una existencia independiente de la mente. El complemento de la existencia de un mundo material que sea independiente de la mente no es un mundo material que sea dependiente de la mente — esa lectura tan parcial del idealismo_-, sino la postulación de un mundo material que no es independiente de la mente. Es una diferencia que deja la puerta abierta a una infinidad de conexiones posibles que no tienen que pasar por una causalidad simple o una producción total de un término por otro: diferentes visiones sobre una misma sustancia, por ejemplo, en el sentido de los "atributos" de Spinoza.

Mediante esta línea de pensamiento, es posible equiparar el concepto, el cual excede la definición cerrada con la que iniciamos, y la riqueza del objeto. Es decir, que a diferencia de las nociones que toman la palabra "atmósfera" y la cargan de sentidos diversos, lo que intento es tomar un objeto de definición fácil ("la capa gaseosa que envuelve a la Tierra”) y seguir, a través de las propias líneas del objeto, los registros y valores que la definición omite, las potencias que dichos registros hacen surgir en diversos modos de existencia, las maneras en las que el objeto se engarza, se articula o incluso se intenta dislocar y arrancarse de otros planos de interpretación, sin dejar de ser ese objeto. Los afectos que se señalan como aquellos que emergen del término "atmósfera" (una atmósfera tensa, por ejemplo) surgen a través de una experiencia humana del término, y las resonancias que dicho término puede ofrecer. Personalmente diría que están plenamente contemplados en el sistema que ofrece Guattari, porque son nodos de formación de una subjetividad; pero así como el ser humano no puede verse como un imperio in imperium (Spi- 
noza, 2005), el objeto atmosférico no se puede separar del resto, configurando lo terrestre como un imperio sub imperium. La atmósfera misma es un nodo de subjetividad que emerge por doquier de maneras insospechadas; $y$ aunque su dimensión de "donante" (donant) sólo puede ser entrevista de manera totalmente especulativa, al menos puede servir — de manera pragmática, siguiendo los efectos de su incorporación en lo actual y su singularización particular en intereses incorpóreos de diversos nodos-, para señalarnos el camino para pensar detenidamente acerca de los diversos Universos de valor que despiertan y movilizan.

\section{V}

Este esbozo ontológico es simplemente eso: la lanzadera de una serie de líneas de exploración para desbrozar un terreno de pensamiento, pero también de decisión.

Nos enfrentamos a un momento de crisis plena. Esta crisis tiene múltiples facetas, lo cual no debería de sorprender dado el aparato ontológico que aquí se desarrolla. La crisis parecería imponernos, comprensiblemente, el problema de una intervención urgente en el registro ambiental y particularmente en el atmosférico. Una manifestación de este impulso por intervenir es la propuesta general de la geoingeniería, es decir, de la modificación dirigida a gran escala de dinámicas terrestres. La propuesta particular que más peso ha tenido en años recientes es la llamada Solar radiation management (SRM), que pretende mediante varios métodos posibles (por ejemplo, la dispersión de aerosoles de compuestos de azufre en la estratósfera) modificar la absorción de una parte de la radiación solar en las capas altas de la atmósfera.

Esta intervención abre un campo de incertidumbre. No se conocen a ciencia cierta los datos de los efectos posibles de esta acción, y previsiblemente, el riesgo mayor de la intervención usualmente se expresa señalando esta información faltante. La posible implementación futura de la geoingeniería promete decidirse alrededor de una serie de datos obtenidos de manera profundamente cibernética: una relación de input y output, entrada de compuestos y salida de efectos. Esta decisión nos ofrece un binarismo absoluto (implementarla o rechazarla), el cual tiende a absorber y anular todas las consideraciones de diversos modos de valor. Dicho de otra manera, al pensar así la decisión se dejará fuera cualquier característica maquínica de heterogeneidad radical, la cual necesariamente informa un grupo ilimitado de Universos de valor distintos; en palabras de Guattari , "los valores son inmanentes a las máquinas" (1996: 72), y el potencial no actualizado de la máquina tiene precisamente el papel no solamente de ser heterogéneo en sus posibilidades de ma- 
nifestación, sino de heterogeneizar sus propios componentes (1996: 54): busca escapar de un colapso alrededor de cualquier rasgo unificador. Tal caracterización me parece estar en consonancia con la propuesta de Massumi (2018: 19): si se puede llamar de alguna manera el valor que deberíamos movilizar que sea consistente con la realidad, este nombre sería el de "creatividad", la invención de nuevas alternativas.

Si se deja fuera la dimensión maquínica en la decisión de la intervención atmosférica, existen varias vías de evolución que puedo entrever. En el peor de los casos, la decisión absorbería todos los Universos de valor en un único dilema generalizado, configurado en una alternativa infernal (Stengers, 2009: 67): una dicotomía de escenarios, en donde una opción parece poco atractiva (la dispersión de aerosoles en las capas altas atmosféricas), pero la alternativa es mucho peor (la marejada de tragedias derivadas del calentamiento global). Las alternativas infernales se producen en situaciones que son "fruto de fabricaciones pacientes, a escala pequeña, de cuidadosas experimentaciones", y a pesar de parecer externamente lógicas, en ellas se mueve una resonancia de amenaza. "Si no elegimos la geoingeniería", parecen decir, "seríamos directa y colectivamente responsables de una tragedia mayúscula”. Su lógica, de esta manera, parece cerrar el ingreso de otros valores. Hay otro caso que me parece entrever: la decisión mostrada como necesariamente derivada de un cálculo cuantitativo. Por ejemplo: ¿Cuántas personas morirían al decidir desplegar un arreglo tecnológico como la SRM? ¿Cuántas personas morirían de no hacerlo? David Keith, líder del "Keith Group” en Harvard, el cual está dedicado a la investigación tecnológica y de política pública para entender la efectividad y los riesgos de la SRM, continuamente reflexiona acerca de los potenciales efectos negativos que implica su propia iniciativa. En una conferencia reciente deja clara su posición:

Éste no es un caso de identificar quién morirá, sino que se trata de cambiar el número de gente que morirá por un montón de enfermedades [asociadas a los efectos contaminantes y de cambio en las dinámicas de ozono en la atmósfera al iniciar la SRM]. El cambio neto de mortalidad [de 130000 muertes adicionales] resulta del hecho de reducir el cambio climático [a través de la SRM]. Los cambios en el clima [causados por la SRM] resultan en una mortalidad adicional significativa; pero yo considero eso como algo moralmente diferente, porque todo el punto de esto es que nos gustaría tener menos cambio climático. (Keith en Harvard Museum of Natural Hisotry, 2019) $)^{7}$

${ }^{7}$ Todos los corchetes son aclaraciones contextuales mías. Los comentarios acerca de la cualidad moral de la geoingeniería comienzan en el minuto 18 '. 
No se pone en duda que el cambio climático sea un problema urgente. Tampoco se puede poner en duda que una decisión debe de ser informada por valores científicos, cuantitativos y corpóreos. Sin embargo, éstos no pueden ser el único punto de apoyo de la decisión, a pesar de que pueda parecer la única vía posible. $\mathrm{Al}$ enfocarse puramente en esos valores, aun presentando de manera honesta el presupuesto calculado de costos y beneficios, se eliminan de la consideración todos los otros Universos de valores; en efecto, valores cuya naturaleza les hace imposible aparecer en un discurso de riesgos y valores cuantitativos, no en poca medida porque su expresión puede ser no discursiva. La presente reflexión es únicamente el esbozo de un panorama ontológico, que nos pueda inspirar a pensar en otros registros, diversos e incluso disonantes. La tarea urgente es tratar de entender cómo es posible articular dichos Universos de valor. No creo que esta tarea produzca un manual de acción: las recomendaciones de Guattari hacen parecer que cualquier constelación de valores producirá una heterogeneidad y, por tanto, una riqueza, de situaciones ilimitadas. Sin embargo, sirva este trabajo como un señalamiento, un punto de partida, para mostrar la necesidad de esa actividad que, según Arendt (1971), parece ser una actividad no natural, alejada de la condición humana, pero que no es nunca privilegio de pocos sino de todos: no la actividad de juzgar, sino de pensar. El pensamiento no juzga porque es un diálogo mudo que trata de invisibles, y como tal se separa, tal vez trabajosa y parcialmente, de la sed de conocimiento científica. Es la habilidad de discernir los valores inmanentes en los eventos, condición necesaria para elaborar un juicio sobre ellos; es la posibilidad de ver la pluralidad en los bordes de los conceptos que a primera vista parecerían determinados en su totalidad.

\section{Referencias bibliográficas}

Alexander, Samuel. (1966). Space, Time, and Deity. Londres: Palgrave Macmillan. Anderson, Ben. (2009). "Affective atmospheres." Emotion, Space and Society, 2(2), 77-81. https://doi.org/10.1016/j.emospa.2009.08.005.

Arendt, Hannah. (1971). "Thinking and moral considerations: a lecture". Social Research, 38(3), 417-446.

Bateson, Gregory. (1999). Steps to an ecology of mind. Chicago: University of Chicago Press.

Cushman-Roisin, Benoit. (2019). Environmental fluid dynamics. Nueva York: John Wiley \& Sons. 
Deleuze, Gilles; Guattari, Félix. (2005). Qu’est-ce que la philosophie? París: Editions de Minuit.

Dolphijn, Rick; van DER TuIn, Iris. (2012). New materialism: interviews \& cartographies. Ann Arbor: Open Humanities Press. 38-47.

DRY, Sarah. (2019). Waters of the World: The story of the scientists who unraveled the mysteries of our oceans, atmosphere, and ice sheets and made the planet whole. Chicago: University of Chicago Press.

Edwards, Paul N. (2010). A vast machine: computer models, climate data, and the politics of global warming. Cambridge, Mass: MIT Press.

Guattari, Félix. (1989). Les Trois écologies. París: Editions Galilée.

Guattari, Félix. (1996). Caosmosis. Buenos Aires: Manantial.

GuAtTARi, Félix. (2011). “On contemporary art”. En Eric Alliez y Andrew Goffey (eds.), The Guattari Effect. Londres; Nueva York: Bloomsbury. 40-55.

Guattari, Félix. (2013). Schizoanalytic cartographies. Londres; Nueva York: Bloomsbury.

Guattari, Félix. (2015). ¿Qué es la ecosofía? Buenos Aires: Cactus.

Haraway, Donna. (2016). "Staying with the trouble: Anthropocene, Capitalocene, Chthulucene”. En Jason Moore (ed.), Anthropocene or Capitalocene? Nature, history and the crisis of capitalism. Oakland, CA: PM Press. 34-76.

Harvard Museum of Natural History. (2019, 12 de diciembre). The peril and promise of solar geoengineering [Archivo de video]. Recuperado de https://youtu. be/xWI2w2F1gMg.

(IPCC) Panel Intergubernamental sobre el Cambio Climático. (2013). Climate Change 2013: The Physical Science Basis. Contribution of Working Group I to the Fifth Assessment Report of the Intergovernmental Panel on Climate Change (T. F. Stocker, D. Qin, G.-K. Plattner, M. Tignor, S.K. Allen, J. Boschung, A. Nauels, Y. Xia, V. Bex y P.M. Midgley, eds.). Cambridge, UK; Nueva York: Cambridge University Press. Recuperado el 29 de junio de 2020 de https:// www.ipcc.ch/site/assets/uploads/2018/02/WG1AR5 all final.pdf.

James, William. (1987). Writings, 1902-1910. Nueva York: Literary Classics of the United States.

James, William. (1996). Essays in radical empiricism. Lincoln: University of Nebraska Press.

Leibniz, Gottfried Wilhelm. (2017). Nuevos ensayos sobre el entendimiento (Juan Ramón Goberna Falque, Trad.). España: Akal.

Lovejoy, Shaun. (2017). "How accurately do we know the temperature of the surface of the Earth?”. Climate Dynamics, (49), 4089-4106. https://doi. org/10.1007/s00382-017-3561-9. 
Massumi, Brian. (2018). 99 theses on the revaluation of value: a postcapitalist manifesto. Minneapolis: University of Minnesota Press.

Morton, Timothy. (2013). Hyperobjects: philosophy and ecology after the end of the world. Minneapolis: University of Minnesota Press.

Schunl, Pierre-Maxime. (1962). Les Stoïciens. Paris: Gallimard.

SpInozA, Benedictus de. (2005). Ética demostrada según el orden geométrico. Madrid: Trotta.

STENGERs, Isabelle. (2009). Au temps des catastrophes: résister à la barbarie qui vient. París: Découverte.

Stengers, Isabelle. (2018). "Science fiction to science studies." En Steven Meyer (ed.), The Cambridge companion to literature and science. Cambridge University Press. 25-42.

STRICKLAnd, Lloyd. (2014). Leibniz's Monadology: a new translation and guide. Edimburgo: Edinburgh University Press.

Whitehead, Alfred North. (1920). The concept of nature. Cambridge: Cambridge University Press. 